\title{
Stable $Q$ analysis on vertical seismic profiling data
}

\author{
Yanghua Wang ${ }^{1}$
}

\begin{abstract}
Vertical seismic profiling (VSP) provides a direct observation of seismic waveforms propagating to various depths within the earth's subsurface. The $Q$ analysis or attenuation $(1 / Q)$ analysis based on direct comparison between individual waveforms at different depths, however, suffers from the problem of instability commonly due to fluctuations inherent in the frequency spectrum of each waveform. To improve the stability, we considered frequency and time variations and conducted $Q$ analysis on an integrated observation. First, we transformed the time- (or depth-) frequency-domain spectrum to a 1D attenuation measurement with respect to a single variable, the product of time and frequency. Although this 1D measurement has a higher signal-to-noise ratio than the $2 \mathrm{D}$ spectrum in the time-frequency domain, it can also be used to further generate a stabilized compensation function. Then, we implemented two $Q$-analysis methods by data fitting (in a least-squares sense) to either the attenuation measurement or the data-driven gain function. These two methods are theoretically consistent and practically robust for conducting $Q$ analysis on field VSP data.
\end{abstract}

\section{INTRODUCTION}

This paper presents robust methods for estimating the quality factor $Q$ from vertical seismic profiling (VSP) data. Stability is a key feature of these $Q$-analysis methods.

Because VSP records the first arrivals at various depths in a vertical borehole, any two waveforms at different depths can be directly compared to measure the attenuation $1 / Q$. It should have a high accuracy in an ideal case because of the high signal-to-noise ratio (S/N) of the VSP first arrivals. However, conventional methods for $Q$ analysis on VSP data based on spectral ratio and centralfrequency shift suffer from the problem of instability. The spectral ratio method directly comparing frequency spectra of two waveforms (Hauge, 1981; Stainsby and Worthington, 1985; Tonn, 1991) often appears unstable in practice because of fluctuations inherent in the frequency spectrum of any individual waveform (White, 1992). The central-frequency shift method estimates $Q$ by observing the shifting quantity of the central or peak frequency from a reference (Quan and Harris, 1997; Liu et al., 1998; Zhang and Ulrych, 2002). This method, again depending upon individual waveforms, is not stable, being strongly subject to the variation of the $\mathrm{S} / \mathrm{N}$ in the spectra. When these methods are applied to surface seismic data, with a much lower S/N than that in VSP data, only limited success would be expected (Dasgupta and Clark, 1998). Nevertheless, fit-forpurpose $Q$ values are used routinely for lithological interpretation (Adam et al., 2009; Reine et al., 2009, 2012). The review papers by Campbell et al. (2005) and Kaderali et al. (2007) amply demonstrate the capacity of VSPs to deliver satisfactory results in an appropriate circumstance.

The instability problem of these conventional methods is due to the frequency-domain working procedure because the analyses on individual waveforms are sensitive to the noise level of the data and to the errors and fluctuations presented in the amplitude spectra. To overcome the problem, Wang (2004) proposes to consider not only the frequency, but also the time in the $Q$ analysis. First, a Gabor transform was performed to a single seismic trace to generate a $2 \mathrm{D}$ spectrum in the time-frequency space. Then, this $2 \mathrm{D}$ spectrum was transformed to a 1D function of a single variable, the product of time and frequency. Rickett (2006) and Reine et al. (2009, 2012) also consider frequency and time simultaneously in the regression for $Q$ determination, by fitting a synthetic surface to a (logarithmic) spectral ratio surface in the time-frequency plane. The latter is composed of spectral ratios of multiple wavelet pairs with different time differences.

The essential concept in Wang (2004) is, however, to define the seismic attenuation function with a single variable $\chi \equiv \omega \tau$, the product of the angular frequency $\omega$ and the time $\tau$. The 1D attenuation measurement $A(\chi)$ was generated by a line integral along a constant $-\chi$ contour over the time-frequency space. Each sample of $A(\chi)$ is in fact an average value along a $\chi$-contour because the

Manuscript received by the Editor 19 July 2013; revised manuscript received 26 February 2014; published online 27 May 2014; corrected version published online 30 June 2014

Imperial College, Department of Earth Science and Engineering, Centre for Reservoir Geophysics, London, UK. E-mail: yanghua.wang@imperial.ac.uk.

(C) 2014 Society of Exploration Geophysicists. All rights reserved. 
$\chi$-contour lines have different lengths and each integral is normalized by its finite length of the contour line. Because it uses integration rather than differentiation, $Q$ analysis based on this 1D attenuation measurement was proved to be more stable, in comparison with that conducted on the time-frequency spectrum directly.

This concept was developed originally for the $Q$ analysis on surface seismic data, akin to the velocity analysis routinely used in reflection seismic signal processing (Wang, 2004). The current paper attempts to extend this method to the $Q$ analysis on VSP first arrivals. Considering VSP first arrival waveforms at different depths associated with different first-arrival times, their superposition would make a single seismic trace varying with time. In other words, these waveforms are a perfect decomposition of a timedomain seismic trace. Fourier transforming the first-arrival waveforms one by one produces a series of frequency spectra, the time-frequency spectrum. Wang (2004) suggests transforming this time-frequency spectrum to the $1 \mathrm{D}$ attenuation measurement by line integral. This integrated observation, rather than the spectra of individual waveforms, is used for $Q$ analysis.

Two methods proposed by Wang (2004) are extended to the $Q$ analysis for VSP data. One is based on the attenuation measurement directly. The other is based on a compensation gain function, derived with stabilization (Wang, 2002, 2003) from the 1D attenuation measurement. The reliability of these two methods is demonstrated using synthetic and field VSP data sets. The estimated $Q$ model is an average $Q$ function that is variable with depth, ranging from the uppermost of the VSP survey down to any depth level. This overall $Q$ model between the source and a receiver can be used for inverse $Q$ filtering to compensate the amplitude and to correct the phase of a VSP first arrival.

\section{STABLE $Q$-ANALYSIS METHODS}

A series of VSP first arrivals recorded at different depths in a vertical borehole can be presented as $A(\tau, \omega)$ in the time-frequency domain. Because the first-arrival times are the physical measurement of waveforms traveling directly from the source to various receivers, the time axis $\tau$ of the time-frequency spectrum is irregularly sampled, due to the variation in the velocity and in the travel path.

The seismic wave with attenuation in the time-frequency domain can be expressed explicitly as

$$
A(\tau, \omega)=A\left(\tau_{a}, \omega\right) \exp \left(-\frac{\omega\left(\tau-\tau_{a}\right)}{2 Q}\right)
$$

where $A\left(\tau_{a}, \omega\right)$ is the amplitude at $\tau_{a}(\geq 0)$ and $Q^{-1}$ is an average attenuation between $\tau_{a}$ and the current time $\tau\left(>\tau_{a}\right)$. This expression does not consider the geometrical spreading and transmission/ reflection effects. For the geometrical spreading, the effect will be explicitly compensated. But the reflection loss is assumed to be negligible, if it is compared to the direct wave propagating through various interfaces.

The $Q$ model is assumed to be frequency independent (Kjartansson, 1979; Wang and Guo, 2004), but could be frequency dependent in practice (Jeng et al., 1999). Defining a single variable $\chi \equiv \omega \tau$, equation 1 can be rewritten as

$$
\bar{A}(\chi) \equiv \frac{A(\chi)}{A\left(\chi_{\mathrm{a}}\right)}=\exp \left(-\frac{\chi-\chi_{\mathrm{a}}}{2 Q}\right)
$$

For the $Q$ analysis, the $2 \mathrm{D}$ spectrum $A(\tau, \omega)$ is transformed to a $1 \mathrm{D}$ attenuation measurement $A(\chi)$ and normalized by a maximum $A\left(\chi_{\mathrm{a}}\right)$. Performing a logarithmic operation on $\bar{A}(\chi)$ leads to a linear form,

$$
y(\chi)=-Q^{-1}\left(\chi-\chi_{\mathrm{a}}\right)
$$

where $y(\chi) \equiv \ln \left[\bar{A}^{2}(\chi)\right]$. Fitting the slope over data samples $\chi \geq \chi_{\mathrm{a}}$ can provide an estimate of the attenuation parameter $Q^{-1}$ as

$$
Q^{-1}=-\frac{\mathbf{x}^{T} \mathbf{y}}{\mathbf{x}^{T} \mathbf{x}},
$$

where $\mathbf{y}$ is the discrete data set $y(\chi)$, for $\chi \geq \chi_{\mathrm{a}}$, and $\mathbf{x}$ is the digitized variable $x=\chi-\chi_{\mathrm{a}}$. This analysis procedure is referred to as an attenuation-based method (Wang, 2004).

Considering the $1 \mathrm{D}$ attenuation measurement $\bar{A}(\chi)$ as the observation, Wang (2004) also proposes a compensation-based $Q$-analysis method. A data-driven gain curve is given as

$$
\Lambda_{\mathrm{d}}(\chi)=\frac{\bar{A}(\chi)+\sigma^{2}}{\bar{A}^{2}(\chi)+\sigma^{2}},
$$

where $\Lambda_{\mathrm{d}}$ with subscript $\mathrm{d}$ indicates that the gain function is derived directly from "data" $\bar{A}(\chi)$, and $\sigma^{2}$ is a stabilization factor. Then, a theoretical compensation function, used in inverse $Q$ filtering (Wang, 2006, 2008), is designed as

$$
\Lambda(\chi, Q)=\frac{\beta(\chi, Q)+\sigma^{2}}{\beta^{2}(\chi, Q)+\sigma^{2}},
$$

where $\beta(\chi, Q)=\exp \left[-\left(\chi-\chi_{a}\right) /(2 Q)\right]$. Fitting the data-driven gain curve with this theoretical function:

$$
J(Q)=\sum_{\chi}\left\|\Lambda_{\mathrm{d}}(\chi)-\Lambda(\chi, Q)\right\|^{2} \rightarrow \min ,
$$

will find an appropriate $Q$ constant.

The stabilization factor $\sigma^{2}$ is linked to the noise level in the data. If including excessively small values of $\bar{A}(\chi)$ that arise from the ambient noise, it would cause large errors in the $Q$ estimation. In practice, one can visually select a support range $\left[\chi_{\mathrm{a}}, \chi_{\mathrm{b}}\right]$ from the $y(\chi)$ curve, which ought to be a straight line within the range. The upper limit $\chi_{\mathrm{b}}$ is set practically corresponding to a threshold cutting off the noise in $\bar{A}(\chi)$.

The compensation-based method exploits the gain function in stabilized inverse $Q$ filtering. It is also conceptually similar to that of Kaderali et al. (2007), who apply inverse $Q$ deconvolution to VSP downgoing direct arrivals, for a range of $Q$ values, and select as a "correct" $Q$ value, which give the most consistent wavelet shape with respect to depth/time, judged from a semblance statistic.

Use of the first-arrival times, rather than the receiver depths, of individual waveforms is certainly attractive because it avoids messing around with raypaths. Then, line integrals can be performed 
over the time-frequency space. For a basic zero-offset VSP, any lateral variation is not considered.

\section{$Q$-ANALYSIS PROCEDURE}

The procedure of VSP $Q$ analysis is demonstrated using a synthetic VSP data set. The accuracy of the analysis can be examined against the exact $Q$ model, and the sensitive parameters can be identified for a reliable $Q$ analysis in practice.

Figure 1a displays the layered velocity model and the layered $Q$ model. The smooth curve in the $Q$ model is the average $Q$ function. The average $Q$ between depth $z_{0}$ and $z_{n}$ is calculated by

$$
Q\left(z_{0}, z_{n}\right)=\left(\sum_{k=1}^{n} \frac{\Delta t_{k}}{Q_{k}}\right)^{-1} \sum_{k=1}^{n} \Delta t_{k}
$$

(Raikes and White, 1984; Wang, 2004), where $\Delta t_{k}$ are the time intervals, $\Delta t_{k}=t\left(z_{k}\right)-t\left(z_{k-1}\right)$, and $t\left(z_{k}\right)$ are the time at various depths, numerically calculated and plotted as a blue dotted line in Figure 1b. The interval time $\Delta t_{k}$ and the horizontal distance $\Delta x_{k}=x\left(z_{k}\right)-x\left(z_{k-1}\right)$ within each layer are calculated numerically as in the following:

$$
\Delta t_{k}=\frac{h_{k}}{V_{k} \sqrt{1-V_{k}^{2} p^{2}}}, \quad \Delta x_{k}=\frac{h_{k} V_{k} p}{\sqrt{1-V_{k}^{2} p^{2}}},
$$

where $h_{k}=z_{k}-z_{k-1}$ is the thickness of the $k$ th layer, $V_{k}$ is its velocity, and $p$ is the ray parameter. Because the interval time $\Delta t_{k}$ is accurately calculated by ray tracing that finds a proper $p$ value, the average $Q$ value takes into account the nonzero offset effect of VSP.

The horizontal offset between the source point and the vertical borehole is $55 \mathrm{~m}$. The source is at depth $15 \mathrm{~m}$, and receivers are placed in the depth range between 15 and $1750 \mathrm{~m}$ with a 5-m interval. Figure $1 \mathrm{~b}$ is the synthetic VSP, generated by a frequencydomain viscoacoustic wave equation:

$$
\left(\nabla^{2}+\frac{\omega^{2}}{c^{2}(\mathbf{x}, \omega)}\right) u(\mathbf{x}, \omega)=0
$$

where $u$ is the plane wave at frequency $\omega$ and $c$ is the complex-valued velocity $c=V_{\mathrm{Re}}+i V_{\mathrm{Im}}$. The real velocity $V_{\mathrm{Re}}$ is the layered velocity given by Figure $1 \mathrm{a}$, and the imaginary velocity $V_{\mathrm{Im}}$ is calculated based on the $Q$ definition $Q^{-1}=-2 V_{\mathrm{Im}} / V_{\mathrm{Re}}$. The source signature is a time-delayed Ricker wavelet with dominant frequency of $30 \mathrm{~Hz}$. The time delay of downgoing waveforms can be found visually by numerically calculating the first-arrival times $t\left(z_{0}, z_{n}\right)=$ $\sum_{k=1}^{n} \Delta t_{k}$ (i.e., the dotted curve in Figure 1b).

The interference of upgoing waves and late downgoing arrivals often affects the seismic spectrum, and it makes accurate $Q$ estimation elusive (White, 1992). Hence, 1D median filtering along the direction parallel to the first-arrival-time curve produces the downgoing wavefield (Figure 2a). For this downgoing wavefield, the geometrical spreading effect must be compensated (Figure $2 b$ ) because the fundamental wave expression 1 does not take this effect into consideration and the $Q$ analysis will be conducted on a wide depth range within which this effect cannot be ignored. Rickett (2006) takes this effect within a short time window as an amplitude scalar to be inverted simultaneously with the interval attenuation effect.
Figure 3 displays the aligned downgoing wavefield, geometrical spreading compensation, and the depth-frequency spectrum. In the latter, the solid white curve indicates the central frequency and two dotted curves define the bandwidth. The central frequency $\left(f_{c}\right)$ and the (half-) bandwidth $\left(f_{b}\right)$ are calculated as (Berkhout, 1984; Barnes, 1993)

$$
f_{c}=\frac{\sum_{f} f A^{2}(f)}{\sum_{f} A^{2}(f)}, \quad f_{b}=\left(\frac{\sum_{f}\left(f-f_{c}\right)^{2} A^{2}(f)}{\sum_{f} A^{2}(f)}\right)^{1 / 2},
$$

where $f$ is the frequency in hertz and $A^{2}(f)$ is a power spectrum.
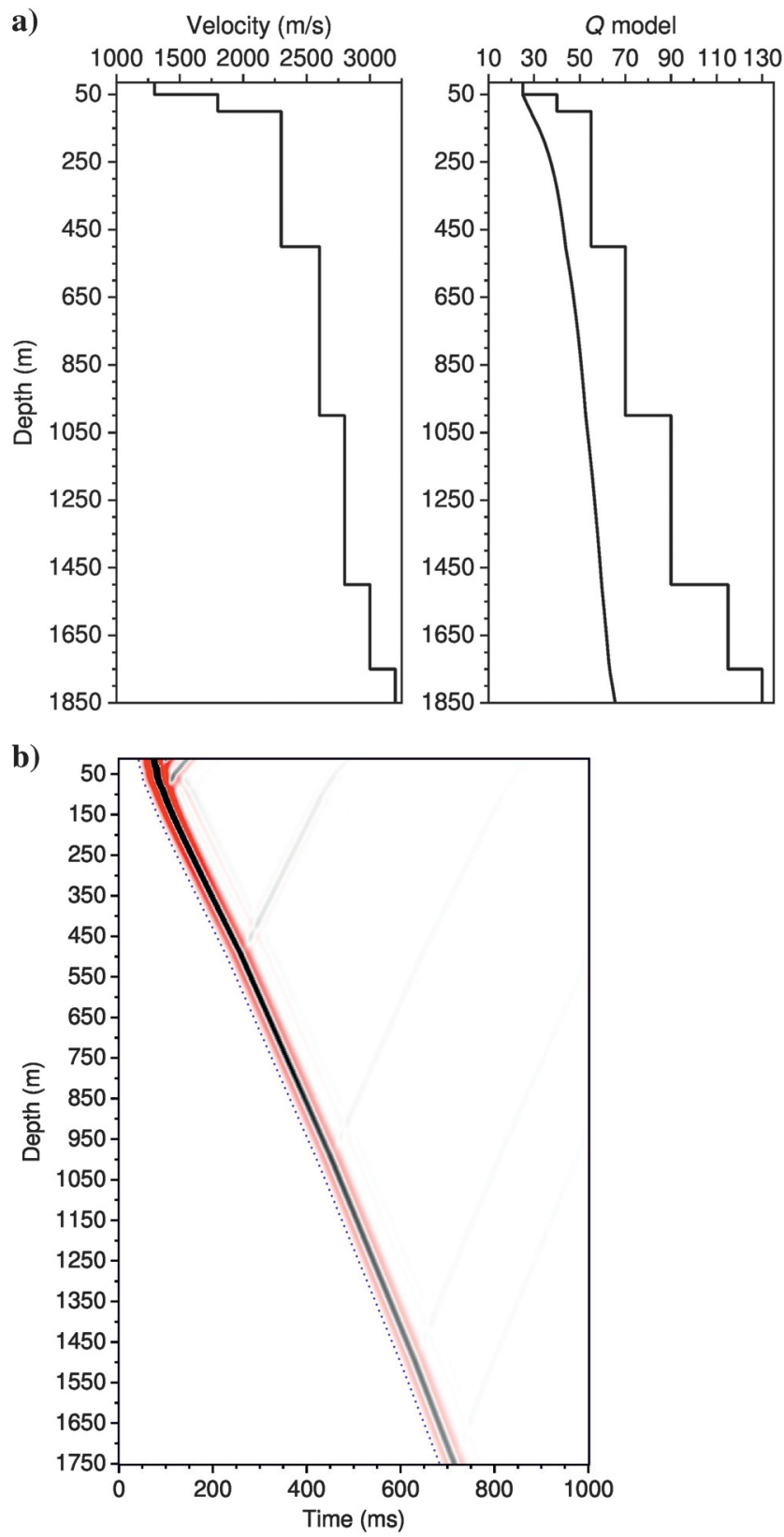

Figure 1. (a) Layered velocity model and layered $Q$ model, in which the smooth curve is the average $Q$ function. (b) VSP synthetic, generated by frequency-domain wave equation with complex-valued velocities. 
In the time-frequency spectrum, the interference of reflections is clearly evidenced at the depths of interfaces (where the arrows point to). At those depths, the derivative of central frequency estimates with respect to the depth will have sharp variations, which could cause the instability problem in conventional $Q$ estimation. For the $Q$-analysis methods presented in this paper, however, this 2D spectrum is transformed to $1 \mathrm{D}$ space with the variable defined as the product of frequency and time $(\chi \equiv \omega \tau)$. In this way, the $Q$ analysis is conducted based on information from all of the first-arrival wave-
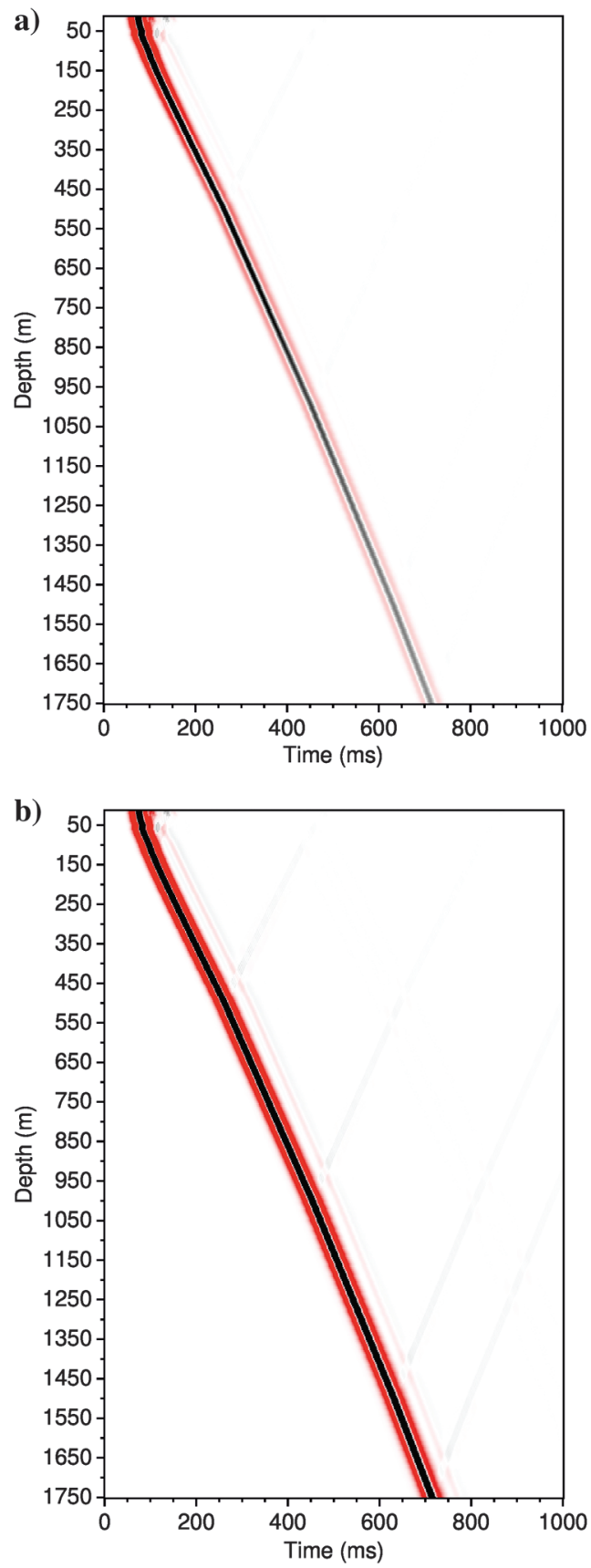

Figure 2. (a) VSP downgoing wavefield, obtained by median filtering. (b) VSP downgoing wavefield, after compensation of geometrical spreading. forms within a depth window rather than directly comparing two individual waveforms.

Figure 4 shows two example depth windows $[15,515]$ and $[15$, $1515] \mathrm{m}$. Each value of the $1 \mathrm{D}$ attenuation function $A(\chi)$ is an average value along the $\chi$-contour because the integral sum along a contour is normalized by the total number of samples along the line. For $Q$ estimates at different depths, the frequency range and the $\chi$ range need to be carefully adjusted.

The procedure for handling different depths recursively is similar to an average-velocity analysis based on a seismic semblance. The 1D attenuation measurement (the top diagrams of Figure 5) is an average observation over an analysis depth window from the uppermost to any current depth. Thus, the estimated $Q$ is an overall value within a depth window. The logarithm of the normalized power spectrum $y(\chi) \equiv \ln \left[\bar{A}^{2}(\chi)\right]$ (solid curves in the middle diagrams of Figure 5) is in a linear trend. For the two examples corresponding to the maximum depths 515 and $1515 \mathrm{~m}$, straight lines (in gray) fit excellently within the ranges $\left[\chi_{\mathrm{a}}, \chi_{\mathrm{b}}\right]=[10,80]$ and $[10,140]$, respectively. The compensation-based method (the bottom diagrams of Figure 5) is also stable and robust in all cases.

Because the $Q$-analysis methods presented in this paper are integral rather than differential-based, the attenuation- and compensation-based methods can produce stable estimates of the average $Q$ values with excellent accuracy (solid curves in Figure 6), in comparison with the theoretical average $Q$ function (the dashed curve in Figure 6).

\section{$Q$ ANALYSIS ON FIELD VSP DATA}

Figure 7 is a field VSP data set in which receivers are placed in a vertical borehole at depths from 50 to $1755 \mathrm{~m}$, with a 5-m depth interval. The horizontal offset between the source and the borehole is $52.3 \mathrm{~m}$. The seismic source is a dynamite type, shot in a 15-mdepth hole. This is a typical land VSP data set, which is used to
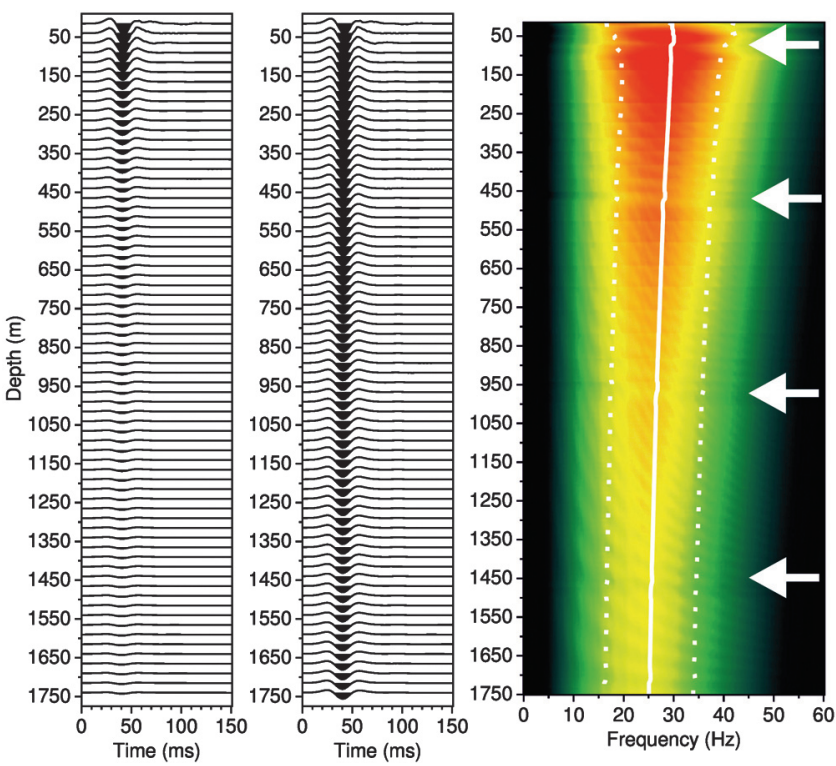

Figure 3. Aligned downgoing wavefield, geometrical spreading compensation, and the depth-frequency spectrum. On the latter, the solid white curve indicates the central frequency, and the two dotted curves define the bandwidth. 
demonstrate the application strategies for the field VSP $Q$ analysis. Subtle and abrupt changes in character at about 300 and $750 \mathrm{~m}$, respectively, in depth might be caused by shot parameters. The integral method can still have a robust $Q$ analysis, and a direct waveform comparison otherwise might not be able to emulate this.

The downgoing wavetrains (Figure 8a), obtained by median filtering, contain waveforms of first arrivals and multiples mostly generated from the free surface. A simple procedure to suppress late arrivals is performed by applying a cosine-square taper (Figure 8b). On the resultant first arrivals, the geometrical divergence effect is also compensated, varying along the receiver depth (Figure $8 \mathrm{c}$ ).

For the selection of tapering parameters, a pragmatic criterion is to eliminate notches from the frequency spectrum. In the top diagram of Figure 9, notches in the spectrum of downgoing wavetrains (without tapering) are the evidence of multiples that follow the first arrival. The lower three curves in Figure 9 are the spectra of downgoing waves with the tapering window $\left[\Delta t_{\mathrm{a}}, \Delta t_{\mathrm{b}}\right]=[80,125],[60,105],[30,75] \mathrm{ms}$, respectively. The last pair is selected as the optimal taper window finally applied to the data, as shown in Figure $8 b$.

Figure 10a selectively shows only the waveforms of VSP first arrivals, aligned at $10 \mathrm{~ms}$, and Figure 10b displays the frequency spectrum of each individual waveform from the entire data set. For this field VSP data set, the central frequency (solid white curve) and the bandwidth (dotted curves) are decreasing, generally along the depth. When generating the attenuation curve with a large depth window, more samples with strong amplitudes (between 10 and $50 \mathrm{~Hz}$ ) will be summed (along a contour as depicted in Figure 4), the normalized sum will have a high $\mathrm{S} / \mathrm{N}$, and $Q$ estimation will be more stable.

Figure 11 shows two examples of $Q$ analysis, corresponding to the maximum depths of 1000 and $1755 \mathrm{~m}$, respectively. The attenuation measurement $A(\chi)$ indicates the coordinate of the maximum at $\chi_{\mathrm{a}}=22$. In both cases, there are excellent attenuation and compensation fittings, although the support ranges are different. They are $\left[\chi_{\mathrm{a}}, \chi_{\mathrm{b}}\right]=[22,175]$ and $[22,545]$, respectively.

Two methods produce similar average- $Q$ functions $Q(z)$, as shown in Figure 12. This is because (1) the attenuation- and compensationbased analyses are consistent in theory, (2) the $\mathrm{S} / \mathrm{N}$ of VSP first arrivals is high in general (compared to surface seismic data and VSP reflection data), and (3) the stability of both methods is similar. For the application to surface seismic traces, to improve the reliability of data fittings, a median filter is often used to mitigate outliers in the attenuation curve $A(\chi)$ and to improve the $\mathrm{S} / \mathrm{N}$ of the "observation," prior to data fitting to either the $y(\chi)$ or $\Lambda_{\mathrm{d}}(\chi)$ function. For the application to VSP first arrivals, this filtering step can be omitted.

In the 2D time-frequency spectrum, seismic reflections appear as localized energy envelopes at different times. The conventional spectral ratio method is conducted based on such a localization feature. The central-frequency shift method might use locally averaged central frequencies, and the shift in between is measured still based on any two localized energy envelopes. However, the $Q$-analysis methods presented here attempt to find a $Q$ value over an integrated
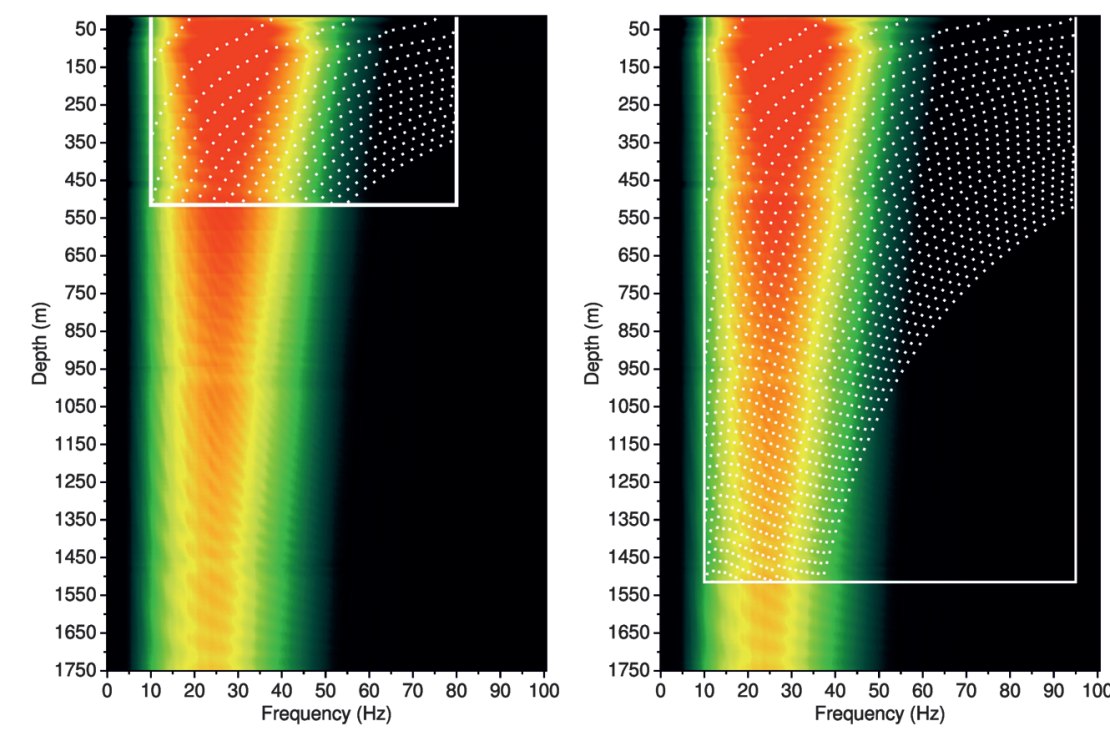

Figure 4. For the $Q$ analysis, the 2D spectrum in the time-frequency domain is transformed to a $1 \mathrm{D}$ attenuation function $A(\chi)$ varying with the single variable $(\chi \equiv \omega \tau)$. Each sample of $A(\chi)$ function is a normalized sum along the $\chi$-contour (a dotted curve) within the predefined analysis depth window and frequency range (a white frame). The left and right panels are two example depth windows $[15,515]$ and $[15,1515] \mathrm{m}$, respectively.
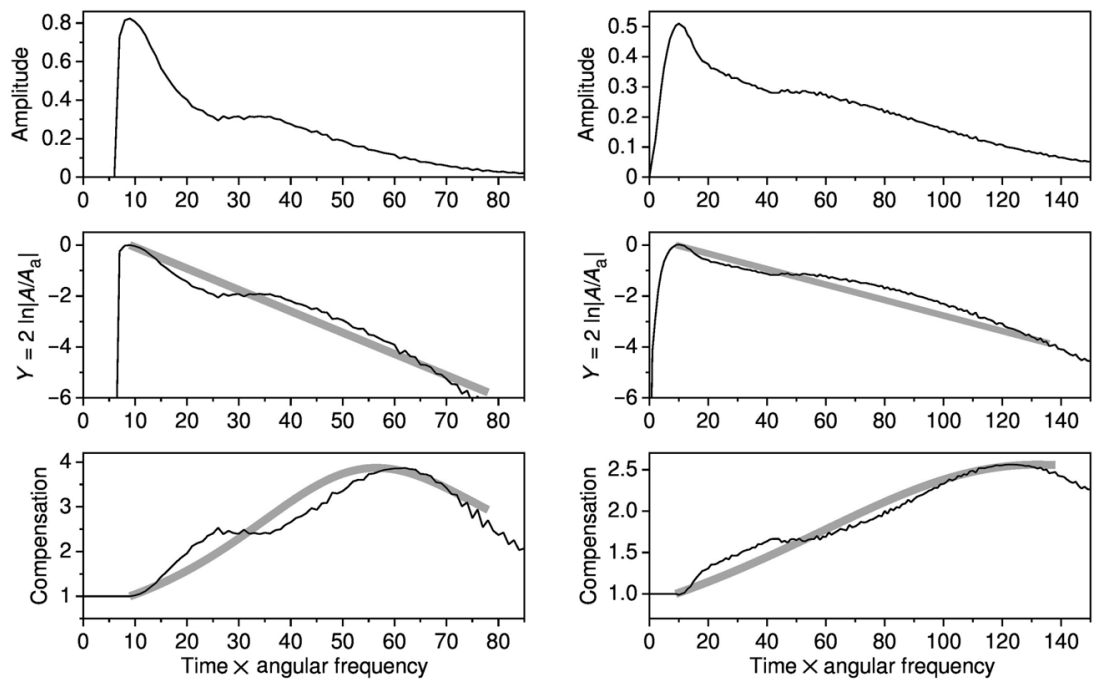

Figure 5. The $Q$ analyses within different depth ranges: $15-515 \mathrm{~m}$ (the left column) and $15-1515 \mathrm{~m}$ (the right column). In each panel, the top diagram is the $1 \mathrm{D}$ attenuation measurement $A(\chi)$, the middle shows the attenuation-based $Q$ analysis, and the bottom shows the compensation-based $Q$ analysis. The solid curves are the observations, and the thick gray curves are theoretical functions. 
measurement $A(\chi)$, rather than the individual waveforms. In this way, stability is achieved for $Q$ analysis on surface seismic and borehole seismic data.
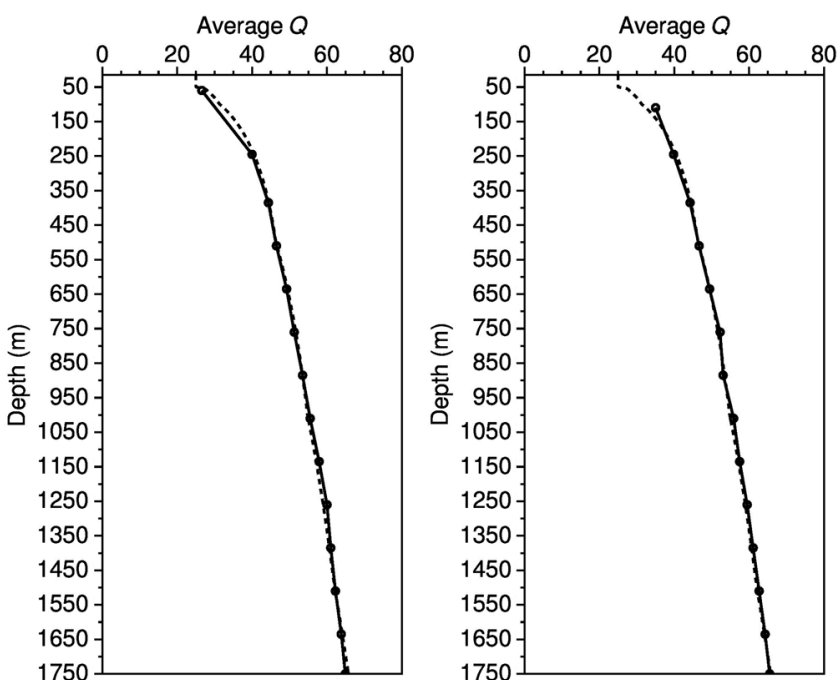

Figure 6. The average $Q$ values (solid curves), estimated using the attenuation- and compensation-based methods. Both methods produce stable estimations, if compared to the theoretical average $Q$ function (dash curves).

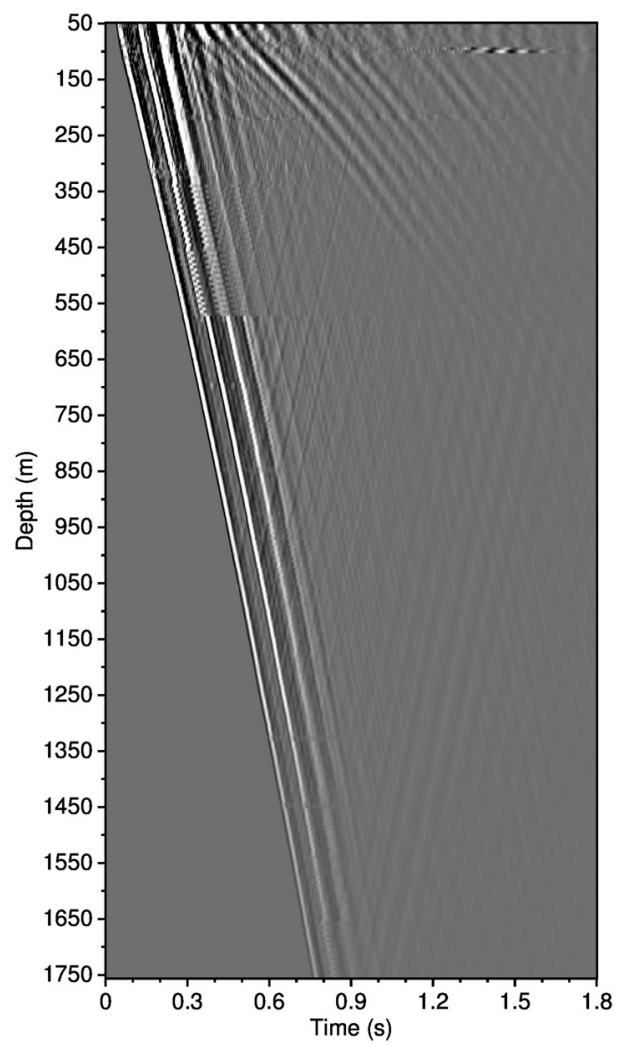

Figure 7. Field VSP data set. Receivers are separated in 5-mdepth interval over the depth range from 50 to $1755 \mathrm{~m}$ in a vertical borehole.
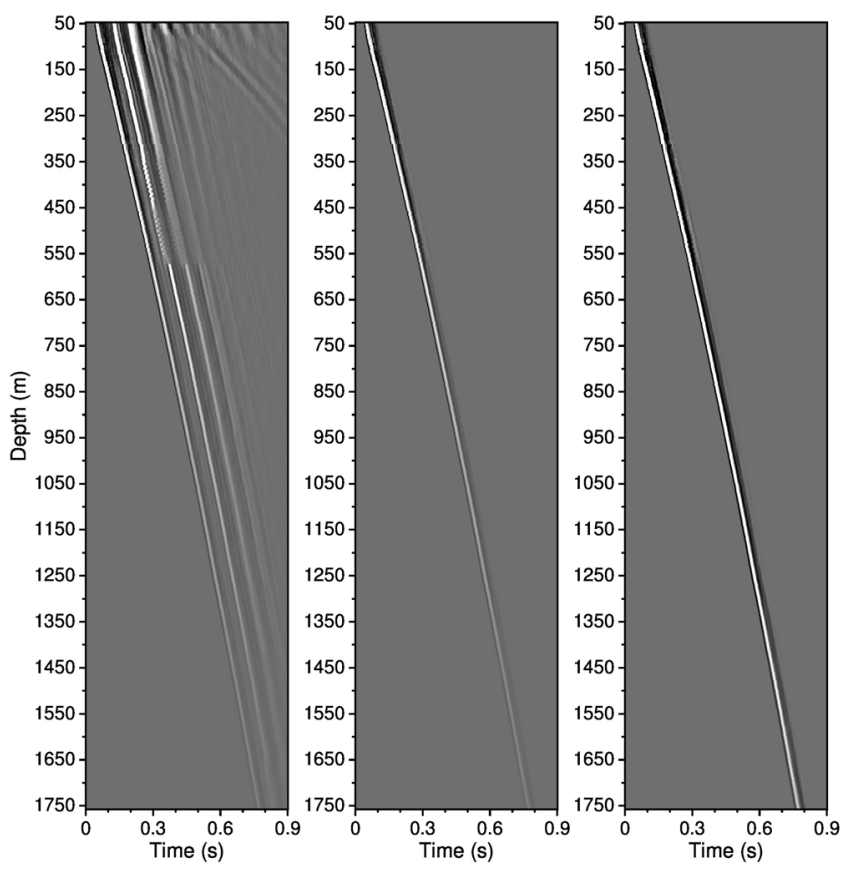

Figure 8. (a) VSP downgoing wavetrains. (b) VSP first arrivals. (c) First arrivals after the compensation of the geometrical divergence effect.
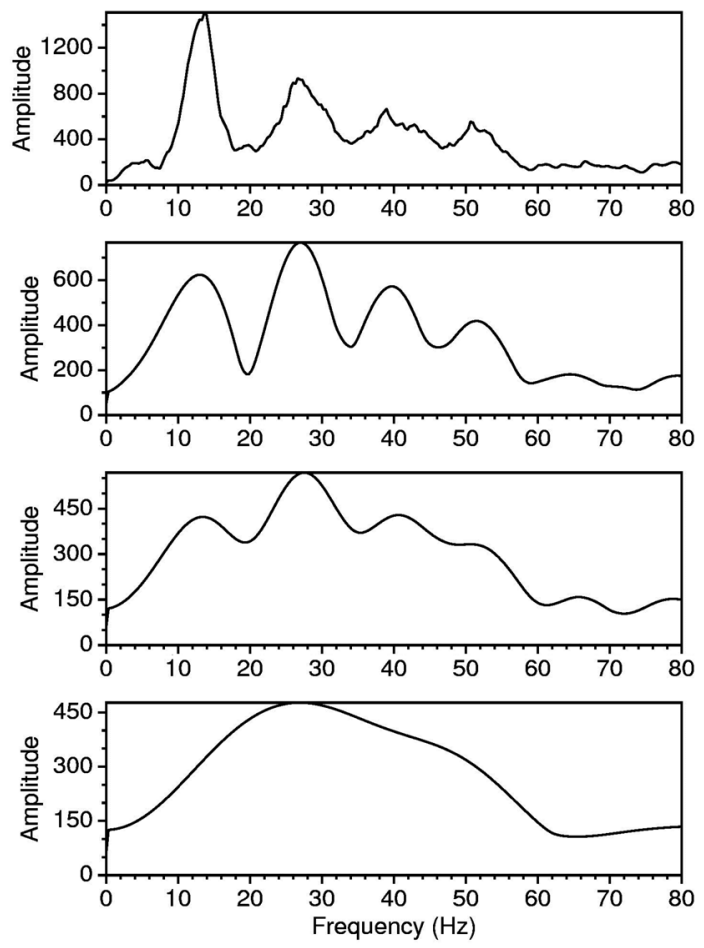

Figure 9. Selection of taper parameters. Notches in the top spectrum of downgoing wavetrains (without tapering) are the evidence of multiples that follow the first arrival. The other three spectra are estimated after applying tapering windows [80, 125], [60, 105], [30, 75] ms, respectively, where the time is the delay time from the first arrival. The basic criterion is to eliminate notches in the frequency spectrum, and the last pair is selected as the optimal window that is finally applied to data (Figure $2 b$ ). 
The only parameters needed are the support region $\left[\chi_{\mathrm{a}}, \chi_{\mathrm{b}}\right]$, which is selected visually based on linearity in the logarithmic power spectrum. This support region physically reflects the limited bandwidth and the finite duration of seismic signals, and thus it is also related to the data noise level $\sigma^{2}$. The latter serves as a stabilization factor in the compensation gain function. Therefore, the two methods are theoretically consistent.

Figure 13 shows the inverse $Q$-filtered result, which can be compared directly to the waveforms and the time-spectrum shown in Figure 10. A stabilized inverse $Q$ filter in the frequency domain is designed as

$$
U(z, \omega)=\tilde{U}(z, \omega) \Lambda(z, \omega) \exp \left[i \omega \tau(z)\left(\left(\frac{\omega}{\omega_{h}}\right)^{-\gamma(z)}-1\right)\right]
$$

where $\tilde{U}(z, \omega)$ and $U(z, \omega)$ are the waveforms before and after inverse $Q$ filtering at depth $z, \gamma(z)=(\pi Q(z))^{-1}, \Lambda(z, \omega)=$ $\left(e^{-\omega \tau(z) /(2 Q(z))}+\sigma^{2}\right) /\left(e^{-\omega \tau(z) / Q(z)}+\sigma^{2}\right), \tau(z)$ is the first-arrival time, and $\omega_{h}$ is a reference frequency. The spectra in Figure 13 show much wider frequency bands and much higher central frequencies than those in Figure 10. Consequently, the first arrivals clearly show narrower waveforms after inverse $Q$ filtering. The stable inverse $Q$ filter does not boost the higher frequencies that were completely attenuated from the original data (Wang, 2002, 2003, 2006). This is why high-frequency components of waveforms in the deep part (depth $>1500 \mathrm{~m}$ ) are not boosted.

Regarding the phase, waveforms at various depths are much more consistent and are aligned along a straight line. However, the first-arrival waveforms at the uppermost part (depth $<150 \mathrm{~m}$ ) differ from most of the VSP record. This is because the data presented here are the vertical components of the VSP record. Those receivers at the shallow part need a proper polarization analysis on three components to reconstruct the direct P-waves.

After inverse $Q$ filtering, the shallow part (depth $<450 \mathrm{~m}$ ) shows an obvious train of multiples. This indicates that the simple tapering process might not be good enough to generate the pure first arrivals. An appropriate treatment could be a global debubbling operation
(Ziolkowski et al., 1980, 1982) with respect to all the VSP downgoing waveforms because this would help to clean up the waveform before muting.
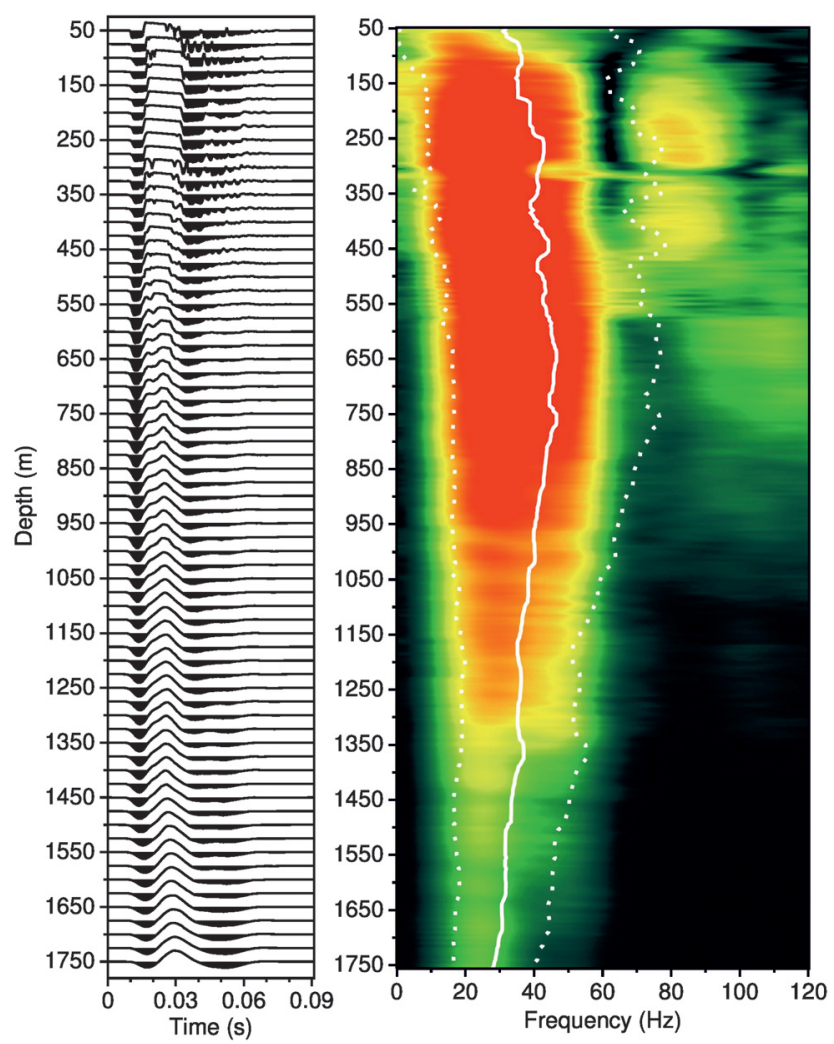

Figure 10. Depth-frequency spectrum. (a) Sparsely selected VSP first arrivals (after the compensation of geometrical divergence), aligned at time $10 \mathrm{~ms}$. (b) The amplitude spectrum of each individual waveform of the entire data set. The solid and the two dotted curves indicate the central frequency and the bandwidth, respectively.
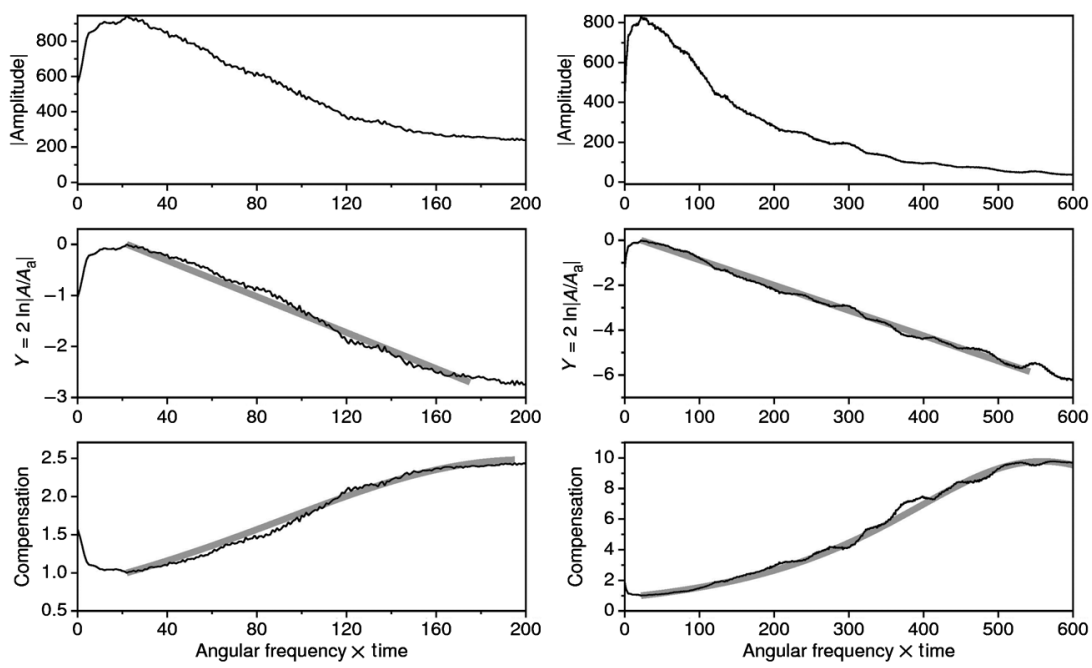

Figure 11. The average- $Q$ analyses within different depth ranges: $50-1000 \mathrm{~m}$ (the left column) and 50-1755 m (the right column). In each panel, the top diagram is the $1 \mathrm{D}$ attenuation measurement $A(\chi)$, the middle shows the attenuation-based $Q$ analysis, and the bottom shows the compensation-based $Q$ analysis. The solid curves are the observations, and the gray curves are theoretical functions. 


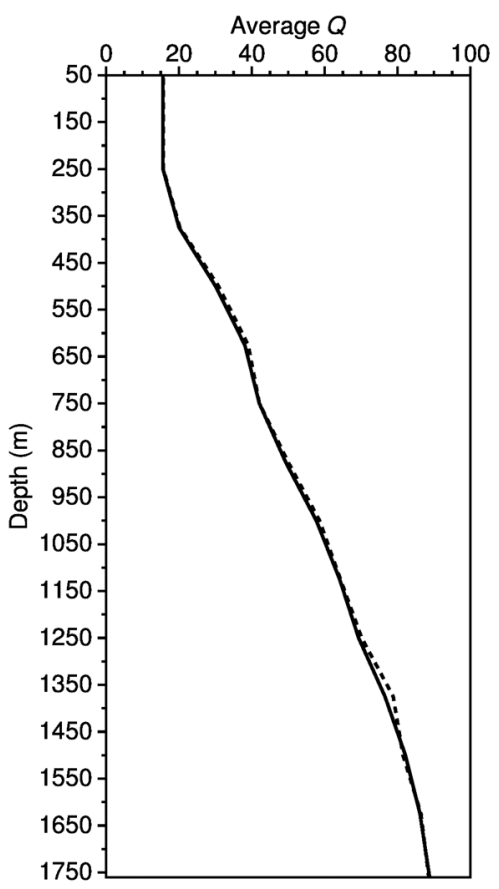

Figure 12. The $Q$ functions estimated by the attenuation-based (dashed curve) and the compensation-based (solid curve) $Q$-analysis methods. Each $Q(z)$ value at receiver depth $z$ is the source-toreceiver overall $Q$.
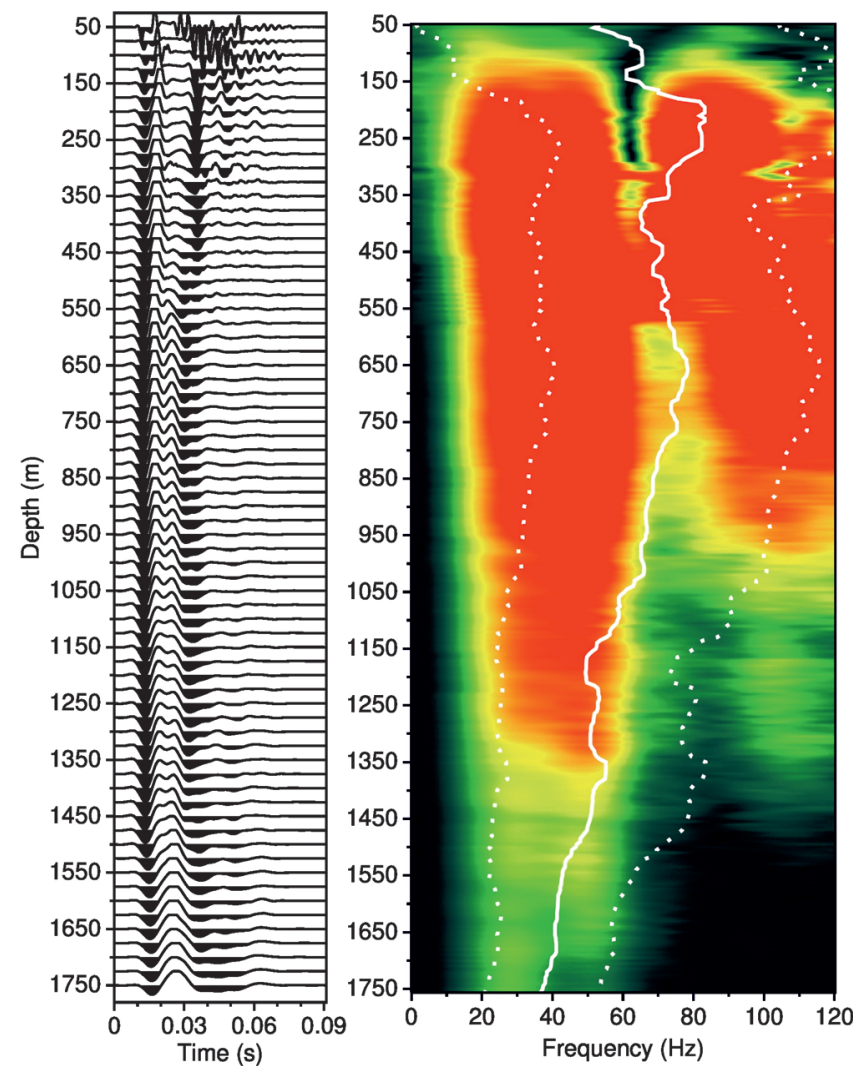

Figure 13. Inverse $Q$-filtered result. (a) Sparsely selected VSP first arrivals. (b) Amplitude spectrum of each individual waveform of the entire data set. The solid and two dotted curves indicate the central frequency and the bandwidth, respectively.

\section{CONCLUSIONS}

For the $Q$ analysis on VSP data, stability can be achieved if the analysis is conducted on an integral type of attenuation measurement in the time-frequency space, rather than on the frequency spectra of individual waveforms. The attenuation measurement is a 1D function of a single variable, the product of frequency and time, and it is obtained by a line integral in the time-frequency space. Because the integral sum is normalized by the line length, the measurement is in fact an average amplitude along the time-frequency contour and, hence it has a higher $\mathrm{S} / \mathrm{N}$ than the time-frequency spectrum. Its (logarithmic) spectrum is then used to estimate a reliable $Q$ value by straight-line fitting. Alternatively, fitting a compensation function in a least-squares sense will also find the $Q$ value. Attenuation- and compensation-based methods are consistent theoretically and practically, as demonstrated by synthetic and field VSP data examples.

For a practical application of VSP $Q$ analysis, two sets of parameters are sensitive. They are the range of frequencies and the support range of the attenuation function. Because VSP first arrivals have a very high $\mathrm{S} / \mathrm{N}$, the compensation-based method produces a result similar to the attenuation-based method, and it does not show much of a significant advantage. Because the gain function is derived with stabilization from the attenuation, the compensation-based method has high expectations in surface seismic $Q$ analysis. Because the measurement is a sum over various depths, the effect of geometrical spreading needs be compensated, although the transmission loss (i.e. reflection) is not considered in this study.

\section{ACKNOWLEDGMENTS}

The author is grateful to the sponsors of the Centre for Reservoir Geophysics, Imperial College London, for supporting this research.

\section{REFERENCES}

Adam, L., M. Batzle, K. T. Lewallen, and K. van Wijk, 2009, Seismic wave attenuation in carbonates: Journal of Geophysical Research, 114, B06208, doi: $10.1029 / 2008 J B 005890$

Barnes, A. E., 1993, Instantaneous spectral bandwidth and dominant frequency with applications to seismic reflection data: Geophysics, 58 419-428, doi: 10.1190/1.1443425.

Berkhout, A. J., 1984, Seismic resolution: Resolving power of acoustical echo techniques: Geophysical Press.

Campbell, A., A. Fryer, and S. Wakeman, 2005, Vertical seismic profiles More than just a corridor stack: The Leading Edge, 24, 694-697, doi: 10 .1190/1.1993259.

Dasgupta, R., and R. A. Clark, 1998, Estimation of $O$ from surface seismic reflection data: Geophysics, 63, 2120-2128, doi: 10.1190/1.1444505.

Hauge, P. S., 1981, Measurements of attenuation from vertical seismic profiles: Geophysics, 46, 1548-1558, doi: 10.1190/1.1441161.

Jeng, Y., J. Y. Tsai, and S. H. Chen, 1999, An improved method of determining near surface $Q$ : Geophysics, 64, 1608-1617, doi: 10.1190/1 .1444665 .

Kaderali, A., M. Jones, and J. Howlett, 2007, White Rose seismic with well data constraints: A case history: The Leading Edge, 26, 742-754, doi: 10 $.1190 / 1.2748491$.

Kjartansson, E., 1979, Constant $Q$-wave propagation and attenuation: Journal of Geophysical Research, 84, 4137-4748, doi: 10.1029/ JB084iB09p04737.

Liu, L., J. W. Lane, and Y. Quan, 1998, Radar attenuation tomography using the centroid frequency downshift method: Journal of Applied Geophysics, 40, 105-116, doi: 10.1016/S0926-9851(98)00024-X.

Quan, Y., and J. M. Harris, 1997, Seismic attenuation tomography using the frequency shift method: Geophysics, 62, 895-905, doi: 10.1190/1 .1444197 .

Raikes, S. A., and R. E. White, 1984, Measurements of earth attenuation from downhole and surface seismic recordings: Geophysical Prospecting, 32, 892-919, doi: 10.1111/j.1365-2478.1984.tb00745.x. 
Reine, C., R. A. Clark, and M. van der Baan, 2012, Robust prestack Qdetermination using surface seismic data, Part 2, 3D case study: Geophysics, 77, no. 1, B1-B10, doi: 10.1190/geo2011-0074.1.

Reine, C., M. van der Baan, and R. A. Clark, 2009, The robustness of seismic attenuation measurements using fixed- and variable-window timefrequency transforms: Geophysics, 74, no. 2, WA123-WA135, doi: 10 $.1190 / 1.3043726$.

Rickett, J., 2006, Integrated estimation of interval-attenuation profiles: Geophysics, 71, no. 4, A19-A23, doi: 10.1190/1.2209722.

Stainsby, S. D., and M. H. Worthington, 1985, $O$ estimation from vertical seismic profile data and anomalous variations in the North Sea: Geophysics, 50, 615-626, doi: 10.1190/1.1441937.

Tonn, R., 1991, The determination of the seismic quality factor $Q$ from VSP data: A comparison of different computational methods: Geophysical Prospecting, 39, 1-27, doi: 10.1111/j.1365-2478.1991.tb00298.x.

Wang, Y., 2002, A stable and efficient approach of inverse $Q$ filtering: Geophysics, 67, 657-663, doi: 10.1190/1.1468627.

Wang, Y., 2003, Quantifying the effectiveness of stabilized inverse $Q$ filtering: Geophysics, 68, 337-345, doi: 10.1190/1.1543219.
Wang, Y., 2004, $Q$ analysis on reflection seismic data: Geophysical Research Letters, 31, L17606, doi: 10.1029/2004GL020572.

Wang, Y., 2006, Inverse $Q$-filter for seismic resolution enhancement: Geophysics, 71, no. 3, V51-V60, doi: 10.1190/1.2192912.

Wang, Y., 2008, Seismic inverse $Q$ filtering: Blackwell Publishing.

Wang, Y., and J. Guo, 2004, Modified Kolsky model for seismic attenuation and dispersion: Journal of Geophysics and Engineering, 1, 187-196, doi: $10.1088 / 1742-2132 / 1 / 3 / 003$

White, R. E., 1992, The accuracy of estimating $Q$ from seismic data: Geophysics, 57, 1508-1511, doi: 10.1190/1.1443218.

Zhang, C., and T. J. Ulrych, 2002, Estimation of quality factors from CMP records: Geophysics, 67, 1542-1547, doi: 10.1190/1.1512799.

Ziolkowski, A., G. Parkes, L. Hatton, and T. Haugland, 1982, The signature of an air-gun array: Computation from near-field measurements including interactions: Geophysics, 47, 1413-1421, doi: 10.1190/1.1441289.

Ziolkowski, A. M., W. E. Lerwill, W. E. March, and L. G. Peardon, 1980, Wavelet deconvolution using the source scaling law: Geophysical Prospecting, 28, 872-901, doi: 10.1111/j.1365-2478.1980.tb01266.x. 